UDC 001.8:330.59]:339

DOI 10.33111/iep.2019.30.03_eng

\title{
Methodological Approaches To The Definition Of A Country's Public Welfare
}

\author{
Ellana Molchanova, \\ KATERYNA KOVTONIUK \\ OLEKSANDR SAVYCH
}

\begin{abstract}
The article describes the main methodological approaches to assessing the national social wealth. The authors believe that evolutionary changes in the global economy have started a new era, which will transform the traditional world economic system. It has been established that in the conditions of transformational restructuring of the world economic system as a whole, as well as national economic systems, in particular, there is a need to change the criteria for assessing their level of economic development. It has been shown that the traditional macroeconomic indicator of the gross national product (GDP), which is still actively used as a measure of national wealth and the leading classification tool of breaking down countries into wealth groups, thus defining the conditions for their cooperation with international organizations, is limited. It is has been proved that monetary indicators are the basis for assessing the level of national wealth of countries, which allows unilaterally estimate the level of their social wealth. Proceeding from the fact that social wealth can be fully estimated taking into account its social component, the effectiveness of national economic systems is analysed on the basis of such models, namely: Pareto optimum and Nash equilibrium. It is proved that the welfare of nation should be considered as the main economic goal of the functioning of national economy. It is established that in the process of evolutionary development of the world economy, understanding of the elements of welfare has changed (replacement of physiological needs with the level of smartphone battery charge and access to WI-FI). It has been proved that economic growth (on the basis of GDP and GDP per capita) does not always lead to progress in the social sphere, which requires the use of new indicators. The article considers the economic efficiency of using social and inclusive development indices that combine economic and social components of social wealth. It is proved that alternative indicators of public wealth assessment are not free from disadvantages, which, on the one hand, are related to the complexity of calculation, and, on the other hand, the lack of a developed methodology for taking into account the whole spectrum of problems in the social sphere and the sphere of environmental protection. It has been established that the imperfection of the existing methodological instrumentarium places in question the universality of new indicators for assessing the level of public welfare. It is predicted that based on the revolutionary type of development of the world economic system, under the influence of rapid development of information technologies, indicators of assessment of the level of social welfare will be modified to meet the needs of the age.
\end{abstract}

\footnotetext{
* This article was translated from its original in Ukrainian.

Molchanova Ellana Yuriivna - Ph.D., Associate Professor, Associate Professor at the Department of International Economics, Deputy Dean at the Faculty of International Economics and Management of Kyiv National Economic University named after Vadym Hetman. Sphere of scientific interests: new economic order, globalization of economic development, global economic integration. E-mail: ellanam@ukr.net

Kovtoniuk Kateryna Volodymyrivna - Ph.D., Associate Professor, Associate Professor at the Department of International Economics, Kyiv National Economic University named after Vadym Hetman. Sphere of scientific interests: European integration, global economic integration, new economic order, digital economy, economic growth. E-mail:k.kovtoniuk@gmail.com

Savych Oleksandr Petrovych - Ph.D., Associate Professor at the Department of Marketing, Kyiv National Economic University named after Vadym Hetman. Sphere of scientific interests: automotive marketing, industrial marketing, marketing management, purchasing marketing, sales management. E-mail: savych@kneu.edu.ua
}

IEP, № 30, (2019) pp. 44-69

(C) Ellana Molchanova, Kateryna Kovtoniuk, Oleksandr Savych, 2019 «All rights reserved» ISSN 1811-9832/2019/№ 1 (30) 
KEYWORDS: social welfare, economic and social well-being, economic growth, gross domestic product, Pareto optimum, Nash equilibrium, social development index, inclusive development index.

\section{Introduction}

Economic growth is the most important characteristic of the country's social production, reflecting the quantitative and qualitative improvement of the social product over a certain period of time. Quantitative expression is reflected in the increase of national production and the growth of economic power of the country ${ }^{2}$, therefore, the macroeconomic indicator of GDP has been country's economic growth criterion for a long time. However, over time, more and more theorists and practitioners draw attention to the impossibility of further using the value of all manufactured end products and services in all areas of the national economic system for consumption, export and accumulation, regardless of the nationality of the used factors of production as an indicator of national prosperity of the country. For example, American politician R. Kennedy said: «We have been evaluating the quality of society on the basis of a simple accumulation of material things for too long and too much. Our growth of domestic product ... If we are to judge on this basis think about air pollution, advertising of cigarettes and the number of ambulances. Count the number of special locks on our doors and prisons for those who break them ...» ${ }^{3}$. High GDP figures hide social problems such as growing inequalities, environmental pollution, lowering the satisfaction of basic human needs, and so on. In addition, there is a strong causal relationship between the impact of factors such as war and environmental / natural disaster on the growth of GDP, driven by rising public expenditures ${ }^{4}$. Thus, the preconditions for changing the paradigm of assessing the country's economic prosperity are formed through the use of new indicators that have a social orientation, but GDP figures are still too early to consider as a rival.

\footnotetext{
${ }^{2}$ Andreeva E. H. Ekonomycheskyi rost. Modely Ekonomycheskoho rosta / E. H. Andreeva, A. N. Sukhova // Omskyi nauchnyi sotsyolohychesky y ekonomycheskye nauky vestnyk. — 2011. — No. 6 (102). - pp. 46-50. [In Russian]

Robert F. Kennedy speeches at the University of Kansas [Electronic resource]. — 18 march 1968. Accessed: https:/www.jfklibrary.org/learn/about-jfk/the-kennedy-family/robert-f-kennedy/robert-f-kennedy-speeches/ remarks-at-the-university-of-kansas-march-18-1968

${ }^{4}$ Rodionov-Zrazhevskiy A. G. Ot epohi valovogo vnutrennego produkta k «ekonomike schastya»/ A. G. RodionovZrazhevskiy // Problemyi sovremennoy ekonomiki. — 2013. — \# 3 (47). — pp. 129-131. [In Russian]
} 


\section{Gross domestic product as a measure of economic well-being of the national economy}

An indicator of GDP was proposed by American economist Simon Kuznets in 1934. The twentieth century, however, became widely used only after the Second World War due to the lack of tools for assessing parameters of the functioning of national economies. At that time, GDP provided an opportunity to quantify the scale of national economic systems in the world, in particular, their real sectors. The assessment was carried out on the basis of comparison of volumes of production of consumer and industrial goods ${ }^{5}$. Against the background of spread of processes of globalization, there is a rapid formation of elements of the new economy, which is characterized by structural transformations in economic systems. These transformations have begun the era of global transit, characterized by the transfer of production from developed countries to developing countries; moving jobs from the sphere of production to the sphere of intellectual services; the transformation of net importing countries of industrial goods into net exporting countries (specializing in the production of high-tech goods and services); the creation of value added in the financial sector due to speculative operations, which is not accompanied by an increase in national welfare and quality of life of the population. Thus, there is a need to justify the expediency of using a traditional indicator, for example, GDP or a new macroeconomic indicator for comparing the economic development of countries. To this end, we will consider new theoretical and methodological approaches that are related to GDP.

A. P. Rumiantsev, V.V. Rokocha and G.M. Klymko conducted an interesting study on different approaches to classification of countries ${ }^{6}$. After analysing the proposed approach, we can determine that the GDP indicator is present in most of the classification indicators of the level of development of countries:

1. Type of interacting national economies in a developed country with a market economy: a) seven countries with the largest volumes of GDP; b) countries of Western Europe; c) countries of North America: USA, Canada and Mexico;

\footnotetext{
${ }^{5}$ Bondarchuk V. Chomu pokaznyk VVP ne vidpovidaie suchasnym potrebam vymiriuvannia ekonomichnoho zrostannia ta shcho maie pryity yomu na zminu [Electronic resource]/ V.Bondarchuk // Analitychna platforma VoxUkraine — access mode: https:/archive.voxukraine.org/2017/01/04/economicgrowth-ua/ [In Ukrainian].

${ }_{6}$ Mizhnarodna ekonomika: pidruchnyk / 「A. P. Rumiantsev, H. N. Klymko, V. V. Rokocha ta in.]. - [Vyd. pererobl. i dopov.]. — Kyiv: Znannia, 2006. - 480 p. [In Ukrainian].
} 
2. The level of income of the country (in calculation of GDP per capita indicated by the methodology of the International Bank for Reconstruction and Development);

3. Country income level (Atlas World Bank Group method: gross national income (GNI) per capita);

4. The level of indebtedness of a group of middle income countries, but high indebtedness: debt to gross national product (GDP).

This classification has certain disadvantages, in particular, the group of developed countries includes countries that have not large volumes of GDP. For example, membership in the European Union automatically provides the country with the status of a developed market economy. In addition, countries with the largest volumes of GDP do not always have the highest GDP per capita, which is more important for determining the level of economic development of the country. This classification can be supplemented by another classification criterion the degree of involvement in the international economic system. According to this classification, countries are divided into closed and open national economic systems characterized by the following equations $\mathrm{YYc}=\mathrm{C}+\mathrm{I}+\mathrm{G}$ and $\mathrm{Yo}=\mathrm{C}+\mathrm{I}+\mathrm{G}+\mathrm{NX}$, respectively. GDP ( $\mathrm{Yc}-$ GDP of the closed economic system, Yo - GDP of the open economic system) is the defining indicator in these macroeconomic equations, which is formed by consumption expenditure (C), investment expenditures (I), government expenditures (G) and net exports (NX) It should be noted that in Western scientific thought, the equation $\mathrm{C}+\mathrm{I}+\mathrm{G}+\mathrm{NX}=\mathrm{AD}$ (e-book with Connect Plus Economics McGrawHill / Irwin) is an expression of aggregate income ${ }^{7}$ rather than GDP. One can call in question the correctness of these interpretations, but the main idea is to understand the changes in the modern paradigm of perceptions of GDP, as the main indicator determining the level of economic development of the country.

Let us turn to the origins of economic theory. What is the effectiveness of national economy? Functioning of any economic system is based on satisfaction of needs ${ }^{8}$. Profit provides the opportunity to meet social needs, that is, profit is a derivative indicator. Consequently, the ultimate goal of obtaining and using profit (capital) should be to meet its own needs, needs of society and increase national welfare in the conditions of resource constraints.

\footnotetext{
${ }_{8}^{7}$ Mc Connell K. Economics / K. McConnell., 2017. — 984 c. - (21). — (Series in Economics).

8 Litvinyuk $A$. A. Motivatsiya i stimulirovanie trudovoy deyatelnosti. Teoriya i praktika: uchebnik dlya bakalavrov [Electronic resource] / A. A. Litvinyuk. - Moskva: Izdatelstvo Yurayt, 2015. - 398 p. - (Seriya: Bakalavr. Akademicheskiy kurs). — Access mode: https://stud.com.ua/34677/menedzhment/motivatsiya_i_stimuly [In Russian].
} 
Since the main advantage of a competitive market system lies in the ability of markets to provide an efficient allocation of limited resources of society, one should focus on explaining the effectiveness of a competitive economy. To explain, we use the concept of Pareto optimum and Nash equilibrium. The concept of effectiveness of a competitive market system is based on the concept of «Pareto efficiency». Pareto efficiency (Pareto optimum) means that resources are optimally distributed if nobody can improve their position without worsening the situation of the other ${ }^{9}$.

Nash equilibrium is the result in which the strategy of each player is the best among the others taken by the rest of players in the strategy game. This definition is based on the fact that none of the players can achieve the most benefit (maximizing the utility function) if the rest of participants firmly adhere to their own line of conduct ${ }^{10}$. It should be noted that the use of the Nash equilibrium concept requires the introduction of the following hypothesis: players cannot agree and leave this point together. That is, Nash equilibrium implies the absence of coalitions of players, which is preconditioned for non-cooperative games. The concept of Nash equilibrium can obviously be used for co-operative games, provided that there are coalitions. But the important fact is that very often the Nash equilibrium takes place also for pure strategies that is, for a one-time game ${ }^{11}$.

These two theories confirm the idea of well-being. However, modern youth have a completely different interpretation of the welfare (Fig. 1). Taking Maslow's theory as a basis, modern youth considers a completely different value as a basis. The first place is not taken by the physiological needs, but the low battery level of the smartphone and access to WI-FI. Modern youth no longer thinks that the basic needs according to the interpretation of Maslow are the basis of all existence. Representatives of the $\mathrm{Y}$ generation have offered to put the Internet in the basis of Maslow's pyramid for several years. Generation Z does not require the Internet, they want to have access to the network anywhere in the world. But there was another problem - battery level. To satisfy the»basic» needs of Z-generation representatives has become a challenge for manufacturers of modern gadgets.

${ }^{9}$ Natsionalna ekonomika: navch. posib. [Electronic resoursel / A. F. Melnyk, A. Iu. Vasina, T. L. Zheliuk, T. M. Popovych; za red. A. F. Melnyk. - K.: Znannia, 2011. - 464 p. - Access mode: //http://pidruchniki.com/1298010841794/ekonomika/kontseptsiyi_efektivnosti_konkurentnoyi_rinkovoyi_ekonomik i [In Ukrainian].

${ }^{10}$ Mochernyi S.V. Ekonomichna teoriia: pidruchnyk [Elektronnyi resurs] / S. V. Mochernyi, M. V. Dovbenko. K.: Akademiia, 2005. - 856 p. - (Alma mater). - Access mode: //http://pidruchniki.com/ekonomika/ rivnovaga nesha [In Ukrainian].

${ }^{1}$ Shyian A. A. Teoriia ihor: osnovy ta zastosuvannia v ekonomitsi ta menedzhmenti. Navchalnyi posibnyk [Elektronnyi resurs]. - Vinnytsia: VNTU, 2009. - 164 p. - Access mode:: http://inrtzp.vntu.edu.ua/ $\mathrm{pmba/stf/teach/books/Theory.pdf} \mathrm{[In} \mathrm{Ukrainian].}$ 
In contrast to the incompatibility of ideas, the perception of theories of generations can be summed up by a comment of the domestic scientist V. Bondarchuk: «Determination of the success of economic systems should be based on indicators of social efficiency and the provision of material and spiritual needs of society. Precisely these groups of indicators, and not the quantitative volume of manufactured goods, will allow an objective assessment of the degree of development of economic systems and societies» ${ }^{12}$.

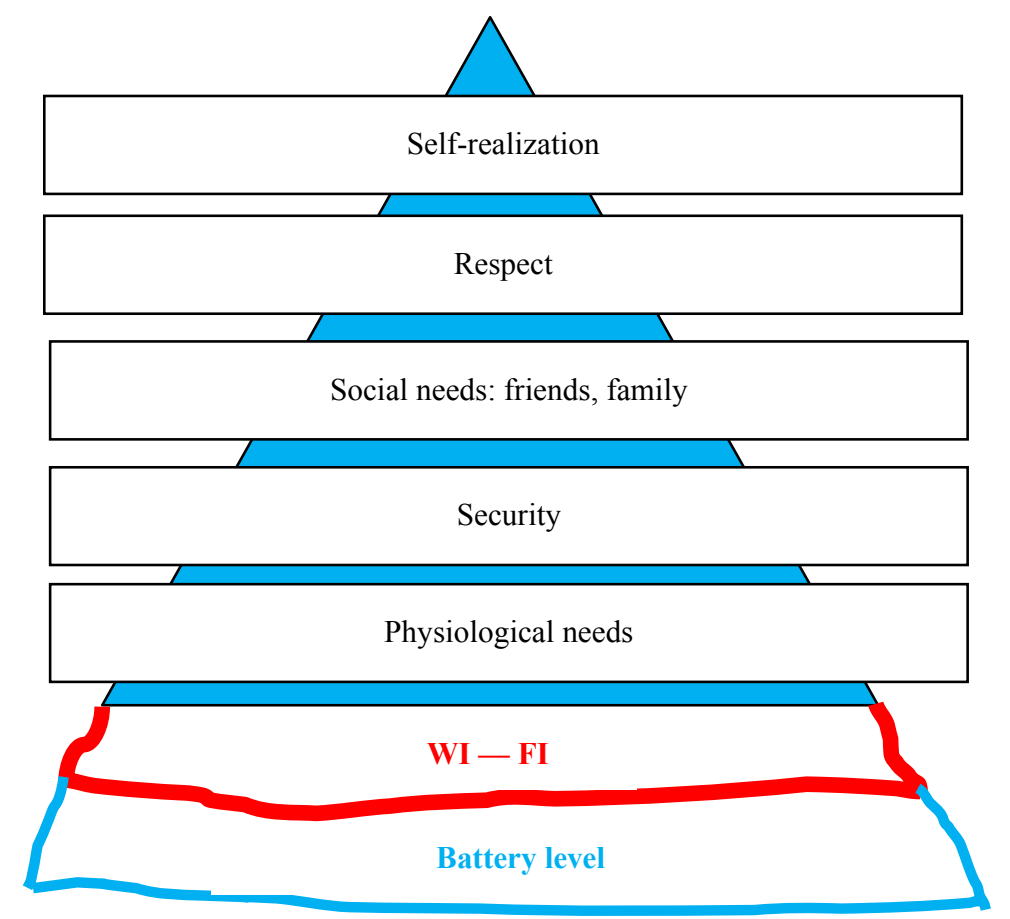

Fig. 1. Interpretation of the basic needs of Maslow theory ${ }^{13}$

So, returning to the essence of GDP, one can not but agree that this indicator does not reflect the qualitative characteristics of the production process of public goods; does not provide any idea of the effectiveness of the use of available resources, on the technology of

12 Bondarchuk V. Chomu pokaznyk VVP ne vidpovidaie suchasnym potrebam vymiriuvannia ekonomichnoho zrostannia ta shcho maie pryity yomu na zminu [Electronic resoursel / V.Bondarchuk // Analitychna platforma VoxUkraine — Access mode: https://archive.voxukraine.org/2017/01/04/economicgrowth-ua/. [In Ukrainian].

Ivaskiv I. Staraya novaya piramida Maslou ili V chyom izmeryaetsya schaste? [Electronic resourse] // Life Academy. - 2018. - Access mode: http://lifeacademy.pearl.pp.ua/staraja-novaja-piramida-maslou-ili-v-chjomizmerjaetsja-schaste.html. [In Russian]. 
production, etc. It is no longer relevant to argue about the ineffectiveness of GDP as the main macroeconomic indicator. Thus, the article of T. Lytovchenko showed that today Nash equilibrium can not provide a macroeconomic equilibrium, because we can not say with certainty that economic relations do not have the incentive to deviate from their current strategy. The second feature is the change in the negotiation process, in particular, the use of «non-cooperative games», based on which the process of gradual study of all possible steps to achieve a compromise, beneficial to all parties of negotiations. But the above is fair only if players of the game fully realize the consequences of their actions. There is uncertainty about future processes due to the rapid development of technological solutions. Part of the problem was analysed by Mark Buchanan in «How Technologies Can Get Rid of Control» at Bloomberg L.P.» ${ }^{14}$. In this article, he makes conclusions based on the scientific work of D. Kusnezov (National Administration of Nuclear Safety) and V. Jones (a former employee of Sandia National Labs). After analysing the probabilities of the development of the world economy on the basis of technological changes, M. Buchanan concluded that under such conditions, the concept of «Nash equilibrium», as an organizational principle of strategic interaction between players, can be considered obsolete ${ }^{15}$.

The idea of strategic equilibrium can not be secured by regulatory mechanisms and instruments. At the present stage of development of the world economy, the efficiency and the object of measurement are important for decision-making. Although there is a direct relationship between economic growth and the well-being of the country's population, the social development rate shows that this rule does not always work. Having the same level of GDP, some countries achieve much higher level of social development than others.

Michael Green, executive director of 'The Social Progress Imperative', emphasized that economic growth does not always lead to progress in the social sphere, while the social development rate shows that if we want to overcome such problems as poverty and inequality, then only one economic growth is not enough.

We will analyse GDP at current prices, as a percentage and GDP per capita to deny or confirm the stated purpose of the study (Fig. 2-4).

\footnotetext{
${ }^{14}$ Buchanan M. How Technology Might Get Out of Control [Electronic resource] / M. Buchanan // Bloomberg. — 2017. — Access mode: https://www.bloomberg.com/opinion/articles/2017-08-15/how-technology-might-get-outof-control

${ }_{5}^{5}$ Lytovchenko T. Epokha rivnovahy Nesha zakinchuietsia? [Electronic resource] / T. Lytovchenko // Chas i podii. - 2017. - No.35. - Access Mode: https://www.chasipodii.net/article/19403/. — Режим доступу до pecypcy: https://www.chasipodii.net/article/19403/ [In Ukrainian].
} 


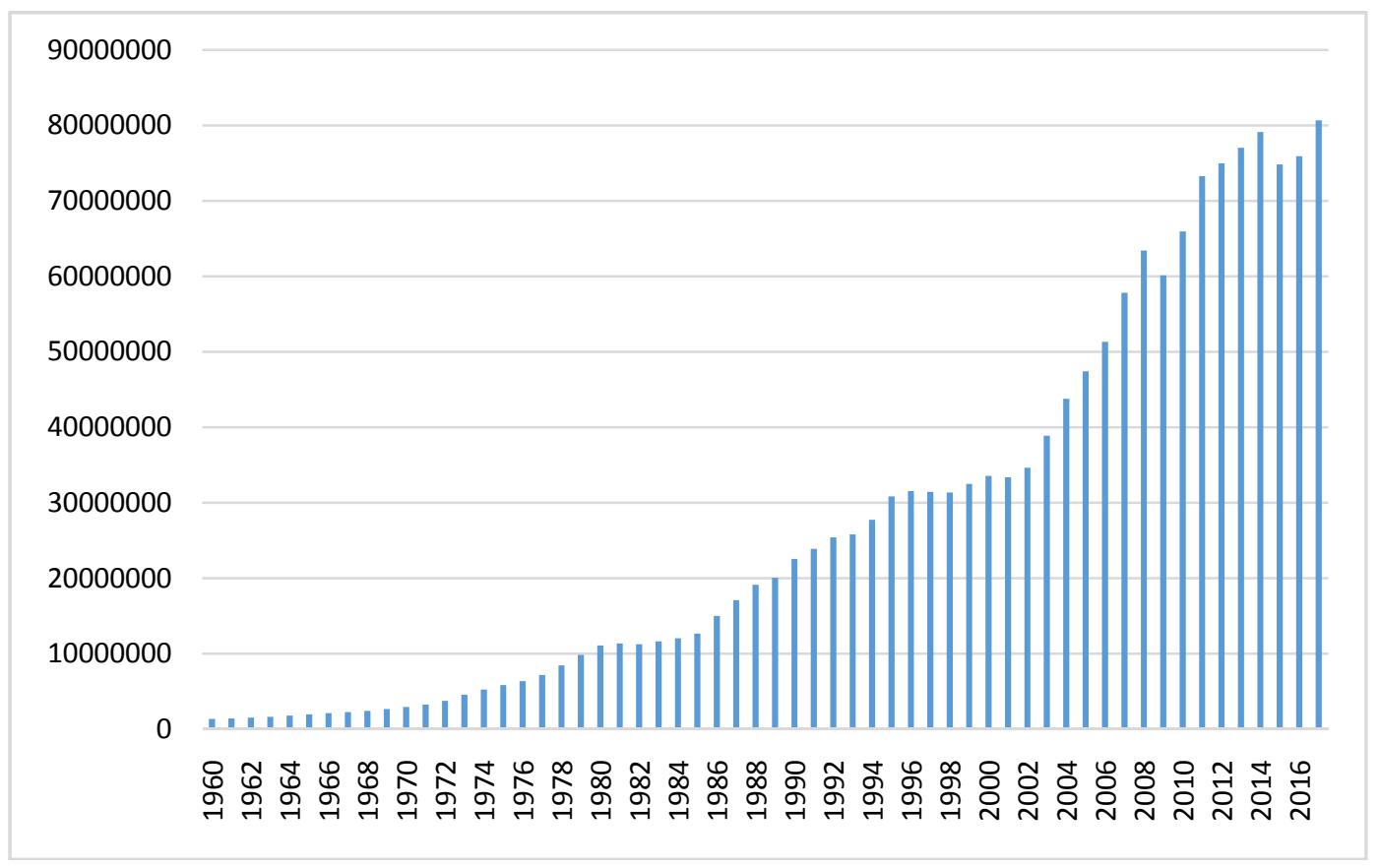

Fig. 2. Gross domestic product in 1960-2017, billion USD ${ }^{16}$

According to the data of Fig. 2, we can follow the positive dynamics of GDP growth in the world. Since 1960 GDP has grown by almost 25 times. If one takes this period, of course, it is positive. However, the insignificant impact of economic cycles is nevertheless traced. If we talk about the crisis of 1974-1975, in addition to reducing GDP in industrialized economies, we do not see it at Fig. 2. The crisis of 19811982 has had a more significant impact on the welfare of countries of the world and slightly slowed the dynamics of world GDP growth. Another slowdown occurred in 1997-1998, mainly in the countries of South-East Asia. In the 90's there was a further minor economic slowdown in the countries. This can be linked to several main reasons: the next division of the world (collapse of the Soviet Union) and the increase in trade, compared with world production. All subsequent periods are characterized by changes in the economic development of world leaders. Thus, the US in the early 2000s shows a decline in economic growth due to the discrepancy between real investment and increased activity of investment in the shares of the IT company in stock exchanges (Table 1).

\footnotetext{
${ }^{16} \mathrm{GDP} /$ World Bank [Electronic Source]. - Access mode: https://data.worldbank.org/indicator/ NY.GDP.MKTP.CD
} 


\begin{tabular}{|c|c|c|c|c|c|c|c|c|c|c|c|c|c|c|c|c|c|c|}
\hline \multirow{4}{*}{\multicolumn{2}{|c|}{$\bar{n}$}} & 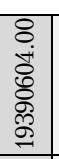 & 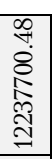 & 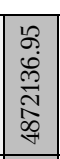 & 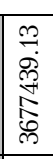 & 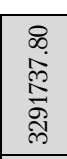 & 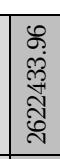 & 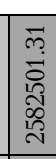 & 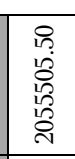 & 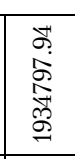 & 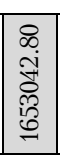 & 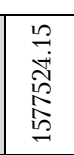 & 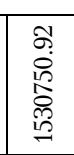 & 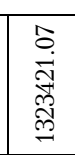 & 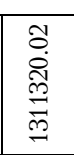 & 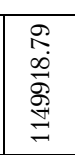 & 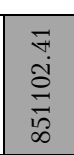 & 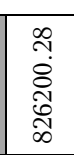 \\
\hline & & $\begin{array}{l}\overleftrightarrow{n} \\
:\end{array}$ & 䔍 & 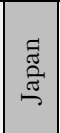 & 胥 & 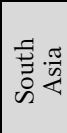 & כ̆ & 总 & $\begin{array}{l}\overline{\bar{N}} \\
\text { صี }\end{array}$ & $\stackrel{\vec{I}}{\mathbb{E}}$ & $\mid$\begin{tabular}{l|}
$\tilde{\pi}$ \\
$\tilde{\Xi}$ \\
$\tilde{J}$ \\
\end{tabular} & 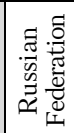 & 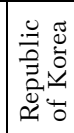 & 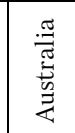 & $\begin{array}{l}\text { 芯 } \\
\text { ڤิ }\end{array}$ & 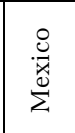 & 总 & 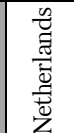 \\
\hline & & 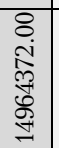 & 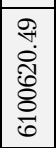 & 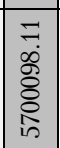 & 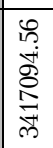 & 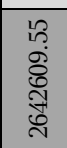 & 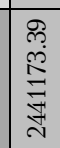 & 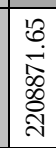 & 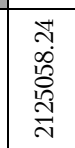 & 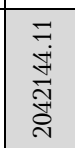 & \begin{tabular}{|l|}
\multirow{7}{*}{} \\
$\stackrel{+}{0}$ \\
$\stackrel{0}{0}$ \\
$\stackrel{2}{0}$ \\
$\stackrel{0}{0}$ \\
\end{tabular} & 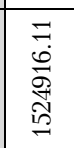 & $\begin{array}{l}\stackrel{1}{2} \\
0 \\
0 \\
0 \\
\stackrel{9}{9} \\
\end{array}$ & 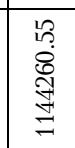 & 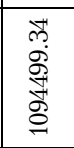 & 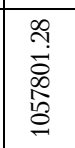 & 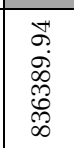 & 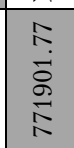 \\
\hline & & $\begin{array}{l}\vec{n} \\
\stackrel{2}{n}\end{array}$ & 䄈 & 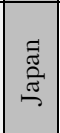 & 鸪 & 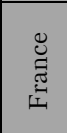 & 히 & $\begin{array}{l}\overline{\bar{N}} \\
\text { 焉 }\end{array}$ & $\stackrel{\vec{J}}{\stackrel{\vec{J}}{*}}$ & 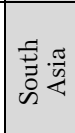 & $\mid$\begin{tabular}{l|}
$\tilde{J}$ \\
$\tilde{\tilde{J}}$ \\
$\tilde{U}$
\end{tabular} & 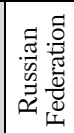 & 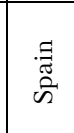 & 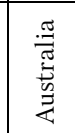 & 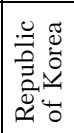 & $\begin{array}{l}\stackrel{8}{\breve{x}} \\
\frac{0}{\Sigma}\end{array}$ & 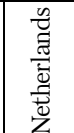 & 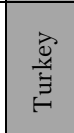 \\
\hline 号 & 8 & 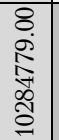 & 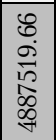 & 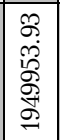 & 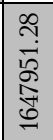 & 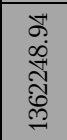 & 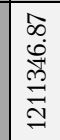 & 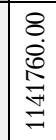 & 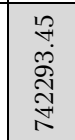 & 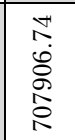 & 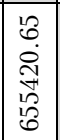 & 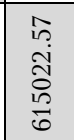 & 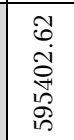 & 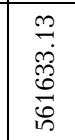 & 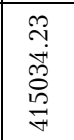 & 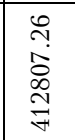 & 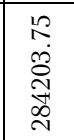 & 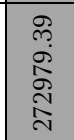 \\
\hline 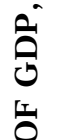 & సั้ & $\begin{array}{l}\overleftrightarrow{n} \\
\stackrel{\omega}{2}\end{array}$ & 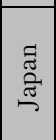 & $\mid \begin{array}{l}\vec{\Xi} \\
\tilde{\Xi} \\
\tilde{\Xi} \\
\end{array}$ & $\breve{~}$ & 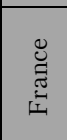 & 苞 & 胥 & $\begin{array}{l}\frac{\pi}{\widetilde{J}} \\
\text { U్ } \\
\widetilde{J}\end{array}$ & 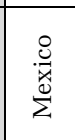 & 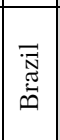 & 毒: & $\begin{array}{l}\text { 离 } \\
\text { की }\end{array}$ & 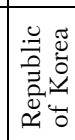 & 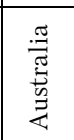 & 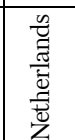 & 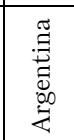 & $\begin{array}{l}\stackrel{\overrightarrow{\mathrm{U}}}{\leftrightarrows} \\
\stackrel{\Xi}{\Xi}\end{array}$ \\
\hline 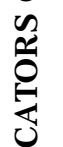 & 8 & 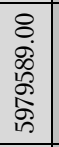 & 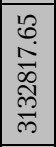 & 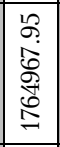 & 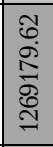 & 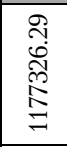 & 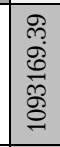 & 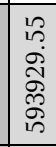 & 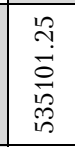 & 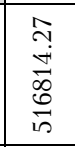 & 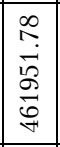 & $\begin{array}{l}8 \\
\stackrel{8}{0} \\
\stackrel{0}{0} \\
\stackrel{\leftrightarrow}{1} \\
\stackrel{\leftrightarrow}{+}\end{array}$ & $\begin{array}{l}\vec{\sigma} \\
\hat{1} \\
\infty \\
0 \\
0 \\
\infty\end{array}$ & 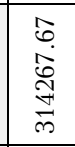 & \begin{tabular}{|l|} 
\\
\\
$\infty$ \\
$\infty$ \\
$\infty$ \\
$\infty$ \\
$\infty$ \\
\end{tabular} & 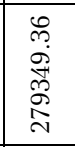 & 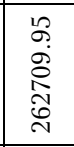 & 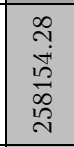 \\
\hline $\begin{array}{l}\underline{z} \\
\underline{Z} \\
\underline{z}\end{array}$ & $\stackrel{9}{\sim}$ & $\begin{array}{l}\overleftrightarrow{n} \\
\stackrel{2}{D}\end{array}$ & 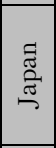 & 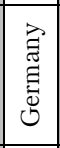 & 总 & $\frac{\vec{\lambda}}{\vec{I}}$ & 光 & 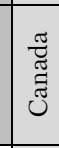 & $\begin{array}{l}\stackrel{\Xi}{\overline{\tilde{N}}} \\
\text { ஸे }\end{array}$ & 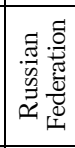 & 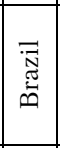 & 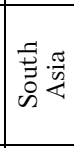 & $\begin{array}{l}\stackrel{\pi}{\Xi} \\
\text { Uี }\end{array}$ & 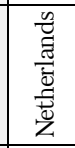 & 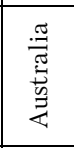 & 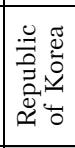 & 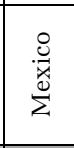 & 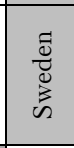 \\
\hline טص & $\varnothing$ & 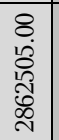 & 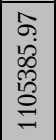 & 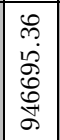 & $\begin{array}{l}\mathcal{7} \\
\infty \\
\infty \\
\stackrel{D}{0} \\
\tilde{D}\end{array}$ & $\begin{array}{l}\overrightarrow{1} \\
\text { Tे } \\
\text { Oे } \\
\text { is }\end{array}$ & 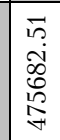 & 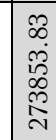 & 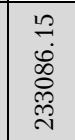 & 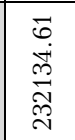 & 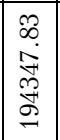 & 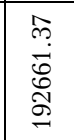 & 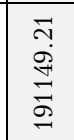 & 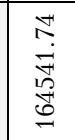 & 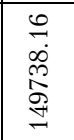 & 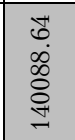 & 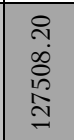 & $\begin{array}{l}\infty \\
\infty \\
\infty \\
0 \\
0 \\
\Xi \\
\Xi\end{array}$ \\
\hline 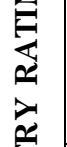 & $\stackrel{.}{ \pm}$ & $\begin{array}{l}\overleftrightarrow{\Delta} \\
\vdots \\
b\end{array}$ & 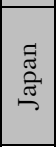 & 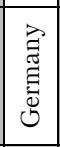 & 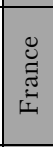 & 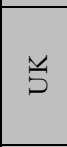 & $\frac{\vec{D}}{\vec{E}}$ & $\begin{array}{l}\frac{\widetilde{\sigma}}{\tilde{U}} \\
\text { Uूँ }\end{array}$ & 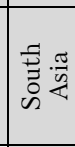 & 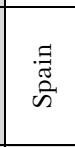 & 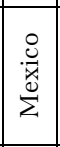 & 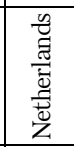 & $\begin{array}{l}\stackrel{\pi}{\Xi} \\
\text { Uี }\end{array}$ & $\begin{array}{l}\vec{z} \cdot \frac{\pi}{3} \\
\bar{z} \\
\tilde{n}\end{array}$ & 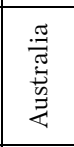 & 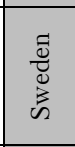 & $\begin{array}{l}\Xi \\
\frac{\Xi}{0} \\
\frac{0}{0} \\
\cong\end{array}$ & 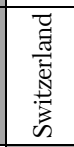 \\
\hline రై & & 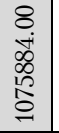 & 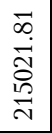 & 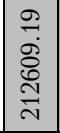 & 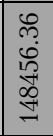 & 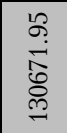 & 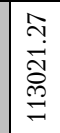 & 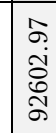 & $\begin{array}{l}\stackrel{\circ}{\circ} \\
\stackrel{\infty}{\infty} \\
\infty \\
\infty\end{array}$ & 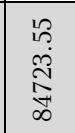 & \begin{tabular}{|l|}
$\mathscr{b}$ \\
$\dot{\vec{d}}$ \\
$\stackrel{\jmath}{\forall}$ \\
\end{tabular} & 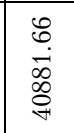 & 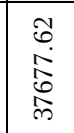 & 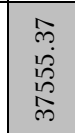 & 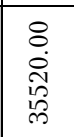 & 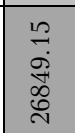 & 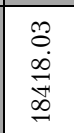 & 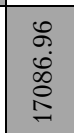 \\
\hline & 5 & $\vec{s}$ & 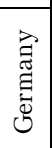 & 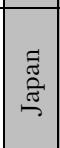 & 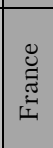 & $\breve{s}$ & $\frac{2}{\vec{E}}$ & 苞 & $\begin{array}{l}\frac{\pi}{\widetilde{J}} \\
\text { Jँ } \\
\tilde{J}\end{array}$ & 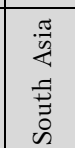 & 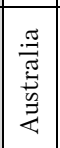 & $\begin{array}{l}\stackrel{\Xi}{\tilde{N}} \\
\text { के }\end{array}$ & 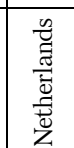 & 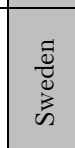 & 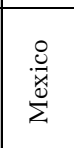 & $\begin{array}{l}\frac{\Xi}{0} \\
\frac{50}{0} \\
\stackrel{0}{0}\end{array}$ & 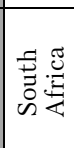 & 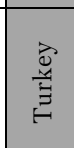 \\
\hline & & 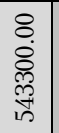 & 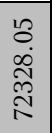 & 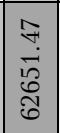 & 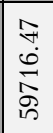 & 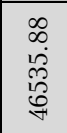 & 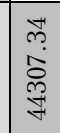 & 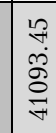 & 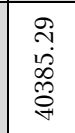 & 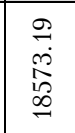 & 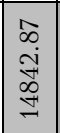 & $\begin{array}{l}\hat{0} \\
1 \\
\delta \\
0 \\
\end{array}$ & $\begin{array}{l}8 \\
\stackrel{0}{0} \\
\stackrel{+}{1} \\
\stackrel{0}{7}\end{array}$ & 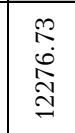 & 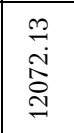 & $\begin{array}{l}\text { N } \\
0 \\
0 \\
0 \\
0 \\
=\end{array}$ & 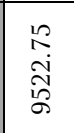 & 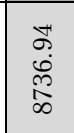 \\
\hline & $\mathscr{2}$ & $\begin{array}{l}\overleftarrow{w} \\
:\end{array}$ & $\breve{5}$ & 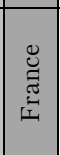 & 苞 & 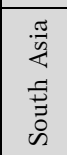 & 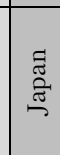 & 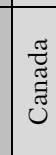 & 画 & 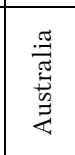 & 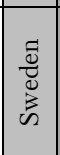 & 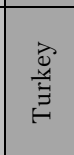 & 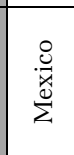 & 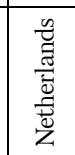 & $\begin{array}{l}\text { 节 } \\
\text { ڤิ }\end{array}$ & 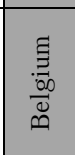 & 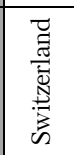 & 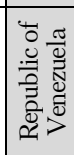 \\
\hline & & - & i & $\dot{m}$ & $\dot{+}$ & is & 0 & $\therefore$ & $\dot{\infty}$ & $\sigma$ & $\dot{0}$ & $\dot{I}$ & $\stackrel{i}{\sim}$ & $\stackrel{m}{\sim}$ & $\dot{I}$ & $\stackrel{2}{2}$ & $\ddot{0}$ & 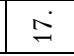 \\
\hline
\end{tabular}




\begin{tabular}{|c|c|c|c|c|c|c|c|c|c|}
\hline \multirow{2}{*}{ ปे } & 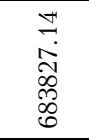 & 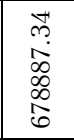 & 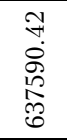 & 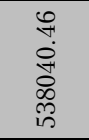 & 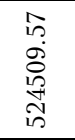 & 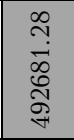 & 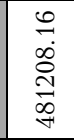 & 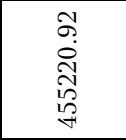 & 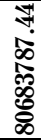 \\
\hline & 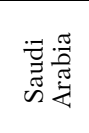 & 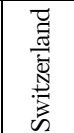 & 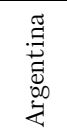 & 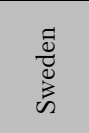 & $\begin{array}{l}\text { 胥 } \\
\stackrel{0}{0} \\
\text { : }\end{array}$ & $\frac{\Xi}{\frac{5}{0}}$ & 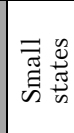 & $\begin{array}{l}\overrightarrow{\widetilde{E}} \\
\frac{\vec{J}}{\bar{J}} \\
\stackrel{F}{F}\end{array}$ & $\begin{array}{l}\overline{7} \\
\vdots \\
3\end{array}$ \\
\hline \multirow{2}{*}{ ฺั } & $\begin{array}{l}\infty \\
\infty \\
\infty \\
\infty \\
\infty \\
\infty \\
\infty \\
\infty\end{array}$ & 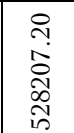 & 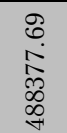 & 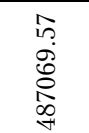 & 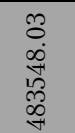 & 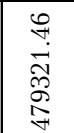 & 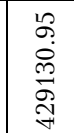 & 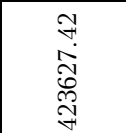 & 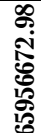 \\
\hline & 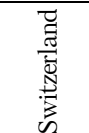 & 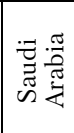 & 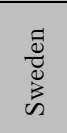 & 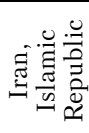 & $\begin{array}{c}\frac{5}{5} \\
\frac{00}{0} \\
\infty\end{array}$ & $\begin{array}{l}\vec{J} \\
\frac{\vec{\sigma}}{0} \\
\stackrel{0}{0}\end{array}$ & $\tilde{E}_{\tilde{Z}}^{\vec{\pi}}$ & 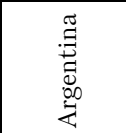 & 훟 \\
\hline \multirow{2}{*}{ ڤ్̀े } & 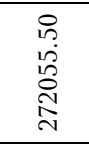 & 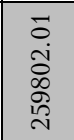 & 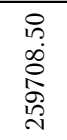 & 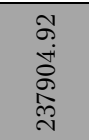 & $\begin{array}{l}\infty \\
\stackrel{\infty}{\circ} \\
\stackrel{8}{\circ} \\
\stackrel{\circ}{2}\end{array}$ & 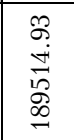 & $\begin{array}{l}8 \\
0 \\
\infty \\
\infty \\
D \\
D\end{array}$ & 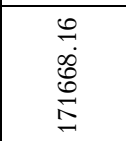 & 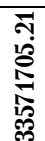 \\
\hline & 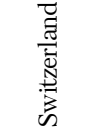 & 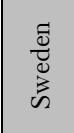 & 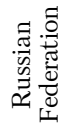 & 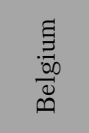 & 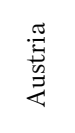 & $\begin{array}{l}\vec{z} \cdot \frac{\pi}{4} \\
\overline{\tilde{n}} \\
\tilde{n} \frac{\pi}{4}\end{array}$ & 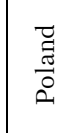 & 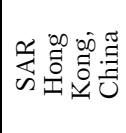 & $\begin{array}{l}\overline{7} \\
\vdots\end{array}$ \\
\hline \multirow{2}{*}{ 兽 } & 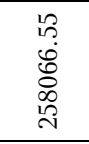 & 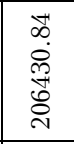 & $\begin{array}{l}1 \\
\infty \\
\infty \\
\infty \\
\infty \\
\infty \\
\\
\end{array}$ & 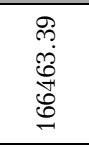 & 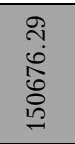 & 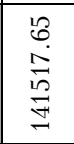 & 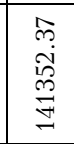 & 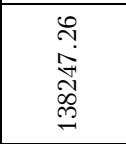 & 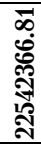 \\
\hline & 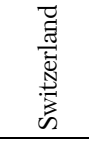 & 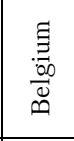 & $\underset{\Xi}{\widetilde{E}}$ & 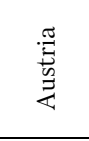 & 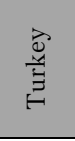 & 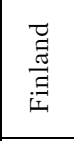 & 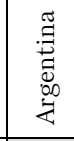 & 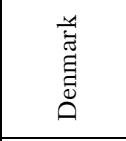 & $\begin{array}{l}7 \overline{0} \\
\vdots \\
3\end{array}$ \\
\hline \multirow{2}{*}{$\stackrel{\infty}{\stackrel{\circ}{\sim}}$} & 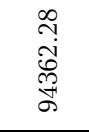 & $\begin{array}{l}\infty \\
\stackrel{\infty}{+} \\
\infty \\
0 \\
\infty \\
\infty\end{array}$ & $\begin{array}{l}\bar{\sigma} \\
\infty \\
\infty \\
\infty \\
\infty \\
\infty\end{array}$ & 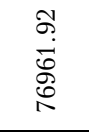 & 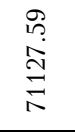 & 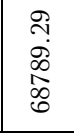 & $\begin{array}{l}1 \\
0 \\
\infty \\
0 \\
0 \\
0 \\
0\end{array}$ & 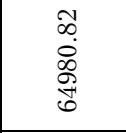 & : \\
\hline & 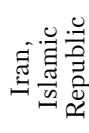 & 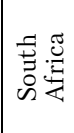 & $\frac{\widetilde{\sigma}}{5}$ & 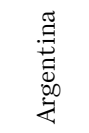 & 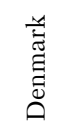 & 总 & 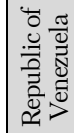 & 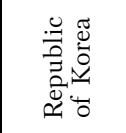 & 훔 \\
\hline \multirow{2}{*}{ 尽 } & 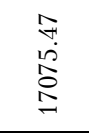 & 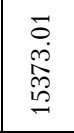 & 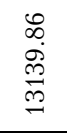 & 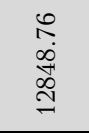 & 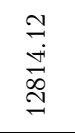 & 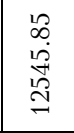 & 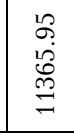 & 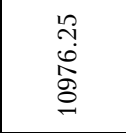 & 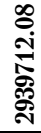 \\
\hline & 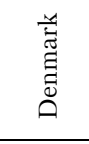 & 莺 & 芯 & 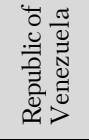 & 密 & 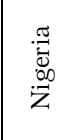 & 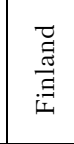 & 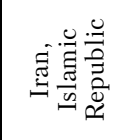 & $\begin{array}{l}\overline{7} \\
\overline{0} \\
3\end{array}$ \\
\hline \multirow{3}{*}{ 兽 } & 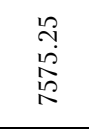 & 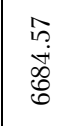 & $\begin{array}{l}\stackrel{8}{0} \\
\stackrel{.}{0} \\
0 \\
0\end{array}$ & 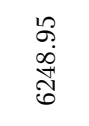 & 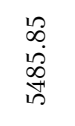 & 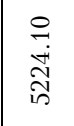 & 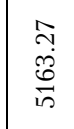 & 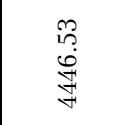 & 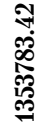 \\
\hline & 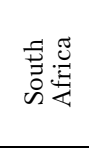 & 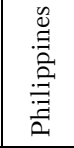 & 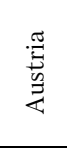 & 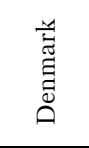 & 恿莺 & 胥 & 总 & $\begin{array}{l}\mathscr{U} \\
\mathscr{\Xi} \\
\tilde{J}\end{array}$ & $\begin{array}{l}\frac{\pi}{0} \\
\dot{0}\end{array}$ \\
\hline & $\stackrel{\infty}{\sim}$ & $\dot{\rho}$ & $\dot{\mathrm{i}}$ & $\dot{\vec{N}}$ & สิ่ & $\stackrel{\sim}{\sim}$ & $\stackrel{\sim}{\sim}$ & ค่ & \\
\hline
\end{tabular}


As we see from table 1, there was a slowdown in Japan's economic growth due to the reduction in purchasing power of the population, and, consequently, the reduction of domestic investment in the country. The US positions remain unchanged, as promoted by the government's policy, which in the 60's recorded annual GDP growth and inflation at $3 \%$ rate, and unemployment at 5-7\%. China's positions show an effective policy of attracting foreign direct investment, establishing appropriate quotas and a successful outsourcing policy.

We can also confidently speak of a consistent policy of the governments of Great Britain, Germany, France, Canada, etc. And Belgium, Sweden, Turkey, and Venezuela, which show a loss of positions every 10 years, have quite different tendency.

Developed industrial countries, as a rule, have annual growth rates of 2$3 \%$ during the period of economic recovery, $1-1,5 \%$ - in the period of insignificant economic crises. Consequently, we can confidently say that in recent times, developing countries have higher rates of economic development (about 6\%), but developed economies - only 2-3\%. So, in 1996 China showed the highest rate of economic development - about $20 \%$.

Another component of the GDP analysis is the nominal GDP (Fig. 3). The difference between the nominal and real GDP lies in the fact that real GDP can only be affected by changes in volumes of manufactured goods. However, the change in the nominal GDP is influenced by the price of products sold.

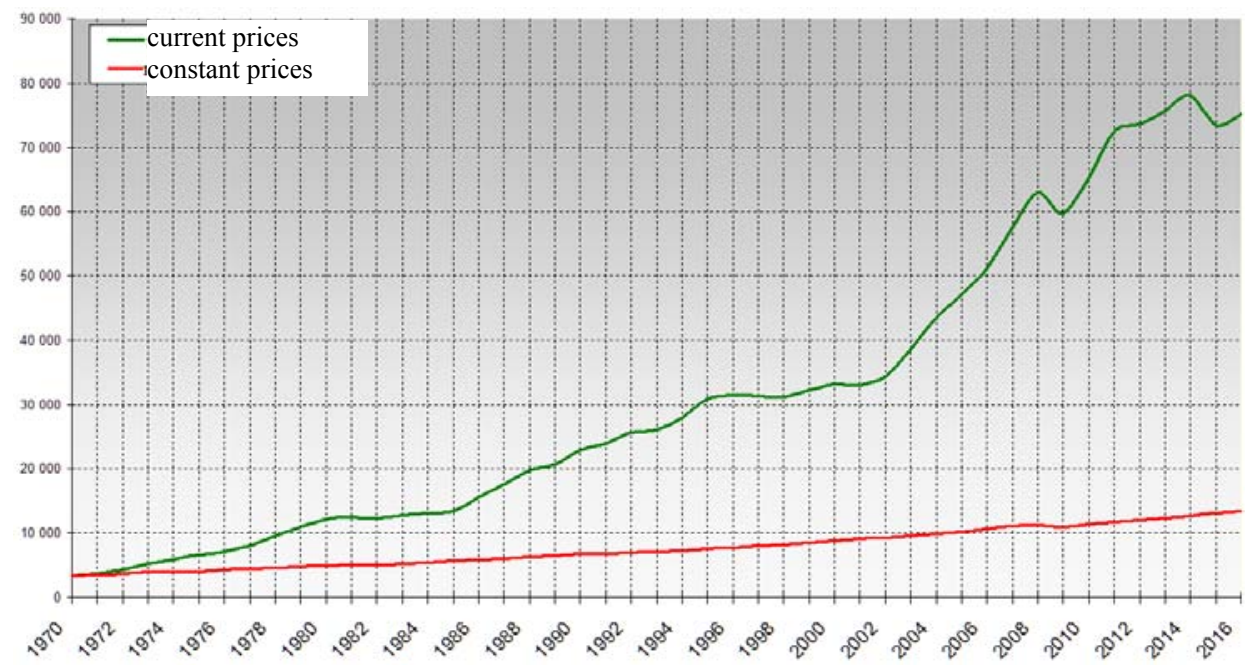

Fig. 3. Nominal GDP of the world ${ }^{1}$

\footnotetext{
${ }^{1}$ Real GDP Growth / International Monetary Fund [Electronic resourcel. - Access mode: http://www.imf.org/ external/datamapper/NGDP_RPCH@WEO/OEMDC/ADVEC/WEOWORLD?year=2018
} 
What gives the nominal GDP of the world countries? It is essentially GDP, but calculated on the basis of market exchange rate, or at the rate set by national governments. In fact, this indicator does not take into account the difference in prices for homogeneous goods and services in different countries. Thus, the nominal value in developed countries will be overestimated in comparison to developing countries.

\section{From the era of gross domestic product to the socio-economic assessment of public welfare}

A comparative assessment of the GDP and GDP by purchasing power parity (PPP) will allow a more complete assessment of the socio-economic components of social growth based on the study of difference in the cost of living in different countries. GDP by purchasing power parity determines the quality of life within the national economy without actual (nominal) comparison based on a single indicator. We face the following problem immediately: when analysing different intervals of economic development of leading countries, we have different forecast data. The first forecast was the $\mathrm{P}$ wC report (Table 2).

Table 2

PREDICTIVE RATING OF COUNTRIES BY AMOUNT OF GDP CALCULATED ON THE BASIS OF PURCHASING POWER PARITY (PPP), IN CONSTANT PRICES OF 2014, IN USD) ${ }^{2}$

\begin{tabular}{|l|l|l|l|l|l|l|}
\hline & \multicolumn{2}{|c|}{2014} & \multicolumn{2}{c|}{2030} & \multicolumn{2}{c|}{2050} \\
\hline $\begin{array}{c}\text { Place } \\
\text { according } \\
\text { to PPP }\end{array}$ & Country & $\begin{array}{c}\text { GDP } \\
\text { according } \\
\text { to PPP }\end{array}$ & Country & $\begin{array}{c}\text { Predictive } \\
\text { rating of } \\
\text { GDP } \\
\text { according } \\
\text { to PPP }\end{array}$ & $\begin{array}{c}\text { Predictive } \\
\text { rating of } \\
\text { GDP } \\
\text { according } \\
\text { to PPP }\end{array}$ \\
\hline 1 & China & 17,632 & China & 36,112 & China & 61,079 \\
\hline 2 & USA & 17,416 & USA & 25,451 & India & 42,205 \\
\hline 3 & India & 7,277 & India & 17,138 & USA & 41,384 \\
\hline 4 & Japan & 4,788 & Japan & 6,006 & Indonesia & 12,210 \\
\hline 5 & Germany & 3,621 & Indonesia & 5,486 & Brazil & 9,164 \\
\hline 6 & Russia & 3,559 & Brazil & 4,996 & Mexico & 8,014 \\
\hline
\end{tabular}

\footnotetext{
${ }^{2}$ Economic forecast «The World in 2050» / $P w C$ [Electronic resource]. — Access mode:
} https://www.pwc.com/ua/uk/press-room/2015/the-world-in-2050.html 
End of the table 2

\begin{tabular}{|c|c|c|c|c|c|c|}
\hline & \multicolumn{2}{|c|}{2014} & \multicolumn{2}{|c|}{2030} & \multicolumn{2}{|c|}{2050} \\
\hline $\begin{array}{c}\text { Place } \\
\text { according } \\
\text { to PPP }\end{array}$ & Country & $\begin{array}{c}\text { GDP } \\
\text { according } \\
\text { to PPP }\end{array}$ & Country & $\begin{array}{l}\text { Predictive } \\
\text { rating of } \\
\text { GDP } \\
\text { according } \\
\text { to PPP }\end{array}$ & Country & $\begin{array}{l}\text { Predictive } \\
\text { rating of } \\
\text { GDP } \\
\text { according } \\
\text { to PPP }\end{array}$ \\
\hline 7 & Brazil & 3,073 & Russia & 4,854 & Japan & 7,914 \\
\hline 8 & France & 2,587 & Germany & 4,590 & Russia & 7,575 \\
\hline 9 & Indonesia & 2,554 & Mexico & 3,985 & Nigeria & 7,345 \\
\hline 10 & UK & 2,435 & $\mathrm{UK}$ & 3,586 & Germany & 6,338 \\
\hline 11 & Mexico & 2,143 & France & 3,418 & $\mathrm{UK}$ & 5,744 \\
\hline 12 & Italy & 2,066 & $\begin{array}{l}\text { Saudi } \\
\text { Arabia }\end{array}$ & 3,212 & $\begin{array}{l}\text { Saudi } \\
\text { Arabia }\end{array}$ & 5,488 \\
\hline 13 & South Korea & 1,790 & South Korea & 2,818 & France & 5,207 \\
\hline 14 & Saudi Arabia & 1,652 & Turkey & 2,714 & Turkey & 5,102 \\
\hline 15 & Canada & 1,579 & Italy & 2,591 & Pakistan & 4,253 \\
\hline 16 & Spain & 1,534 & Nigeria & 2,566 & Egypt & 4,239 \\
\hline 17 & Turkey & 1,512 & Canada & 2,219 & $\begin{array}{l}\text { South } \\
\text { Korea }\end{array}$ & 4,142 \\
\hline 18 & Iran & 1,284 & Spain & 2,175 & Italy & 3,617 \\
\hline 19 & Australia & 1,100 & Iran & 1,914 & Canada & 3,583 \\
\hline 20 & Nigeria & 1,058 & Egypt & 1,854 & Philippines & 3,516 \\
\hline
\end{tabular}

The report is based on a projected analysis of future trends by 2050 , namely, the role of economic counteragents (the loss of the role of leaders by industrialized countries - North America, Western Europe and Japan), despite the projected slowdown in China's growth (approximately after 2020). The $\mathrm{PwC}$ projected for the period until 2050 for the 32 largest countries in the world, accounting to $84 \%$ of world GDP. 
The key conclusions of the $\mathrm{PWC}_{\mathrm{WC}}$ Report «World in $2050 »^{3}$ are:

1) China will undoubtedly become the largest economy by 2030 , but in the long run, the growth rate of the Chinese economy is likely to be at the level of the global average. The growth rate of the Chinese economy will slow down considerably after 2020 as a result of the following factors: aging of the country's population, lower investment returns (China is currently showing high growth rates of investment) and the need to develop innovative solutions more actively to increase labour productivity, rather than copying existing production technologies. China will follow the general trend that has been observed in the past in fast-growing countries such as Japan and South Korea, where high growth rates have declined to worldwide average level over time;

2) Compared to Brazil and Russia, the economy of Colombia and Poland will grow at faster pace over the analysed period until 2050. Indonesia, Mexico, and Nigeria may displace Britain and France out of the top ten. The Philippines, Vietnam and Malaysia will also significantly improve their position in the rating;

3) By 2050 India can compete with the USA for the second place. India has potential to maintain a higher rate of growth of its economy over a longer period and bring its GDP to 10 trillion USD according to purchasing power parity (PPP) approximately around 2020, and according to market exchange rates up to 2035. However, the realization of this potential depends on the consistent implementation of institutional reforms, targeting investments in infrastructure development, and raising the level of education of every segment of population;

4) in the period from 2014 to 2050 , the average growth rate of the world economy is predicted at a level slightly above $3 \%$ per year. As a result, by 2037 the world GDP will double, and by 2050 - it will increase almost three times. However, after 2020, the growth rate of the global economy is likely to slow down under the influence of such factors: lowering the pace of economic growth of China, first and foremost; keeping the moderate growth rates of countries with high GDP growth rates in the long run; slowing down the growth rate of the population of working age in the world.

Let's consider another forecast of GDP according to PPP made by the International Monetary Fund. The calculations were based on data of 2018 (Table 3).

\footnotetext{
${ }^{3}$ Economic forecast «The World in 2050» / $P w C$ [Electronic resource]. - Access mode: https://www.pwc.com/ua/uk/press-room/2015/the-world-in-2050.html
} 
Table 3

PREDICTIVE RATING OF COUNTRIES BY AMOUNT OF GDP CALCULATED ON THE BASIS OF PURCHASING POWER PARITY (PPP), IN CONSTANT PRICES OF 2018, IN USD) ${ }^{4}$

\begin{tabular}{|c|c|c|c|c|c|c|}
\hline & 2018 & $\%$ & place & 2023 & place & PPP \\
\hline USA & $20,412.870$ & 23.3 & 1 & 24,537 & 1 & 1.000 \\
\hline China & $14,092.514$ & 16.1 & 2 & 21,574 & 2 & 1.791 \\
\hline Japan & $5,167.051$ & 5.90 & 3 & 5,962 & 3 & 1.088 \\
\hline Germany & $4,211.635$ & 4.81 & 4 & 5,272 & 4 & 1.039 \\
\hline UK & $2,936.286$ & 3.36 & 5 & 3,477 & 7 & 1.031 \\
\hline France & $2,925.096$ & 3.34 & 6 & 3,586 & 6 & 1.012 \\
\hline India & $2,848.231$ & 3.25 & 7 & 4,663 & 5 & 3.646 \\
\hline Italy & $2,181.970$ & 2.49 & 8 & 2,554 & 9 & 1.100 \\
\hline Brazil & $2,138.918$ & 2.44 & 9 & 2,717 & 8 & 1.584 \\
\hline Canada & $1,798.512$ & 2.06 & 10 & 2,434 & 10 & 1.027 \\
\hline Russia & $1,719.900$ & 1.97 & 11 & 1,974 & 12 & 2.424 \\
\hline Korea & $1,693.246$ & 1.94 & 12 & 2,155 & 11 & 1.263 \\
\hline Spain & $1,506.439$ & 1.72 & 13 & 1,875 & 14 & 1.237 \\
\hline Australia & $1,500.256$ & 1.71 & 14 & 1,958 & 13 & 0.875 \\
\hline Mexico & $1,212.831$ & 1.39 & 15 & 1,586 & 15 & 2.120 \\
\hline Indonesia & $1,074.966$ & 1.23 & 16 & 1,549 & 16 & 3.249 \\
\hline Netherlands & 945.327 & 1.08 & 17 & 1,168 & 18 & 1.023 \\
\hline Turkey & 909.885 & 1.04 & 18 & 1,224 & 17 & 2.550 \\
\hline Saudi Arabia & 748.003 & 0.855 & 19 & 866.0 & 21 & 2.466 \\
\hline Switzerland & 741.688 & 0.848 & 20 & 929.3 & 19 & 0.730 \\
\hline World & 87,505 & & & 114,353 & & 1.543 \\
\hline
\end{tabular}

Source: developed by authors on the basis of information available at http://statisticstimes.com/economy / gdp-nominal-vs-gdp-ppp.php

${ }^{4}$ Comparison of Nominal and Real GDP and GDP per capita / Statistics Times [Electronic resource]. — Access mode: http://statisticstimes.com/economy/gdp-nominal-vs-gdp-ppp.php 
The data in Table 3 contains the predicted GDP of 192 countries at current prices expressed in USD in 2018 and 2023. The GDP in 2018 is expected at the level of 87.51 trillion USD, and PPP - at the level of 134.98 trillion USD. The indicator of GDP is 1,54 times more than PPP in comparison with the nominal index. 174 economies out of 192, have higher value of PPP, and 17 have higher index of nominal value. For the United States, both values are identical. The United States and China remain the world's largest economies in terms of GDP, while Tuvalu is the smallest. As considering nominal value, 16 economies received GDP more than 1 trillion USD, 64 -over 100 billion USD, 178 - more than 1 billion USD. The top five economies account for about $53.51 \%$ of GDP. The top 20 economies account for more than $80 \%$ of GDP. 92 smallest economies make only $1 \%$ of world wealth, and the 155 lowest make only $10 \% .^{5}$

Analysing the last column of Table 3 (GDP per PPP), we can draw the following conclusions: 85 countries received PCS more than 100 billion dollars, and 182 - more than 1 billion dollars. The five leading economies in the world account for $48.92 \%$ of the world's wealth, with 10 leading economies accounting for more than $61 \%$ and the largest 20 countries reach over $75.8 \%$. The 82 smallest economies have only $1 \%$ of the world's wealth, and the lowest 150 have only $10 \%$.

Consequently, apart from the differences in the calculation of GDP and GDP according to PPP, there are many differences in the ratings of economies. Uzbekistan has the highest index in the PPP rating compared to its nominal rank. He ranked 33rd place out of 96 in nominal terms of GDP and 63th in the GDP rating according to PPP. The following are Sudan (+25), Egypt $(+24)$, Myanmar $(+22)$. Iceland is the weakest country in the GDP ranking according to PPP (-45), Luxembourg (-32), Denmark (-25), Papua New Guinea (-22), Malta $(-22) .15$ economies have the same place in both methods.

Having examined the foreseen GDP development rating, we will analyse the genesis of the main macroeconomic indicators (Table 4) and we will draw conclusions about the reliability of the above-mentioned forecast.

As it can be seen from Table 4 , the constant GDP relative to prices in 1970 increased from USD 3,398.7 billion to USD $13,487.4$ billion in 2016. Growth amounted to USD $10,088.7$ billion or 3.97 times. GDP per capita шт constant prices relative to 1970 increased from USD 921 to USD 1,823 in 2016. Growth amounted to USD 902 billion or 1.98 time. This means that the consumer's capacity of one inhabitant of the planet has grown almost once in 46 years.

5 World Economic Outlook. Cyclical Upswing, Structural Change [Electronic resource] // International Monetary Fund. - 2018. - Accessed: https://www.imf.org/en/Publications/WEO/Issues/2018/03/20/worldeconomic-outlook-april-2018 
Table 4

MACROECONOMIC INDICATORS OF THE LEADING COUNTRIES OF THE WORLD, 1970-2016

\begin{tabular}{|c|c|c|c|c|c|c|c|}
\hline \multirow[b]{2}{*}{ Year } & \multirow[b]{2}{*}{$\begin{array}{l}\text { Population, } \\
\text { people }\end{array}$} & \multirow[b]{2}{*}{$\begin{array}{l}\text { Population } \\
\text { growth }\end{array}$} & \multicolumn{2}{|c|}{ Current prices } & \multicolumn{3}{|c|}{ Constant prices 1970} \\
\hline & & & $\begin{array}{c}\text { GDP, } \\
\text { billions } \\
\text { of USD }\end{array}$ & $\begin{array}{c}\text { GDP per } \\
\text { capita, } \\
\text { USD }\end{array}$ & $\begin{array}{l}\text { GDP, } \\
\text { billions } \\
\text { of USD }\end{array}$ & $\begin{array}{c}\text { GDP } \\
\text { growth, } \\
\%\end{array}$ & $\begin{array}{c}\text { GDP per } \\
\text { capita, } \\
\text { USD }\end{array}$ \\
\hline 1970 & $3,692,492,000$ & - & 3,399 & 921 & 3,399 & - & 921 \\
\hline 1971 & $3,706,457,821$ & 2.05 & 3,742 & 994 & 3,542 & 4.22 & 956 \\
\hline 1972 & $3,781,718,408$ & 2.03 & 4,304 & 1,121 & 3,735 & 5.45 & 988 \\
\hline 1973 & $3,857,446,385$ & 2 & 5,236 & 1,337 & 3,972 & 6.34 & 1,030 \\
\hline 1974 & $393,259,749$ & 1.97 & 5,931 & 1,485 & 4,048 & 1.01 & 1,029 \\
\hline 1975 & $4,008,830,123$ & 1.92 & 6,631 & 1,630 & 4,071 & 0.81 & 1,018 \\
\hline 1976 & $4,083,945,282$ & 1.87 & 7,160 & 1,728 & 4,289 & 5.09 & 1,050 \\
\hline 1977 & $4,158,595,800$ & 1.83 & 8,054 & 1,909 & 4,462 & 4.03 & 1,073 \\
\hline 1978 & $4,233,141,702$ & 1.79 & 9,542 & 2,223 & 4,658 & 4.39 & 1,100 \\
\hline 1979 & $4,308,250,508$ & 1.77 & 10,986 & 2,515 & 4,847 & 4.06 & 1,125 \\
\hline 1980 & $4,384,610,765$ & 1.77 & 12,265 & 2,759 & 4,935 & 1.82 & 1,126 \\
\hline 1981 & $4,462,520,455$ & 1.78 & 12,515 & 2,765 & 5,032 & 1.97 & 1,128 \\
\hline 1982 & $4,541,925,121$ & 1.78 & 12,424 & 2,697 & 5,059 & 0.54 & 1,114 \\
\hline 1983 & $4,622,966,917$ & 1.78 & 12,745 & 12,718 & 5,205 & 2.87 & 1,126 \\
\hline 1984 & $4,706,050,232$ & 1.8 & 13,108 & 2,746 & 5,443 & 4.59 & 1,157 \\
\hline 1985 & $4,791,491,780$ & 1.82 & 13,475 & 2,772 & 5,644 & 3.69 & 1,178 \\
\hline 1986 & $4,898,800,655$ & 2.24 & 15,636 & 3,159 & 5,820 & 3.11 & 1,188 \\
\hline 1987 & $4,989,444,582$ & 1.85 & 17,670 & 3,505 & 6,030 & 3.6 & 1,208 \\
\hline 1988 & $5,081,783,861$ & 1.85 & 19,750 & 3,845 & 6,307 & 4.59 & 1,241 \\
\hline 1989 & $5,174,442,999$ & 1.82 & 20,649 & 3,949 & 6,548 & 3.84 & 1,266 \\
\hline 1990 & $5,266,008,555$ & 1.77 & 22,881 & 4,303 & 6,744 & 2.98 & 1,281 \\
\hline 1991 & $5,355,775,164$ & 1.7 & 24,002 & 4,440 & 6,827 & 1.24 & 1,275 \\
\hline 1992 & $5,443,546,997$ & 1.64 & 25,673 & 4,675 & 6,959 & 1.93 & 1,278 \\
\hline 1993 & $5,529,445,444$ & 1.58 & 26,153 & 4,691 & 7,066 & 1.54 & 1,278 \\
\hline 1994 & $5,613,585,107$ & 1.52 & 27,900 & 4,931 & 7,287 & 3.12 & 1,298 \\
\hline
\end{tabular}

\footnotetext{
${ }^{6}$ Countries by Proiected GDP / Statistics Times [Electronic resource]. — Access mode: http://statisticstimes.com/economy/countries-by-projected-gdp.php
} 
End of the table 4

\begin{tabular}{|c|c|c|c|c|c|c|c|}
\hline \multirow[b]{2}{*}{ Year } & \multirow[b]{2}{*}{$\begin{array}{l}\text { Population, } \\
\text { people }\end{array}$} & \multirow[b]{2}{*}{$\begin{array}{l}\text { Population } \\
\text { growth }\end{array}$} & \multicolumn{2}{|c|}{ Current prices } & \multicolumn{3}{|c|}{ Constant prices 1970} \\
\hline & & & $\begin{array}{l}\text { GDP, } \\
\text { billions } \\
\text { of USD }\end{array}$ & $\begin{array}{c}\text { GDP per } \\
\text { capita, } \\
\text { USD }\end{array}$ & $\begin{array}{l}\text { GDP, } \\
\text { billions } \\
\text { of USD }\end{array}$ & $\begin{array}{c}\text { GDP } \\
\text { growth, } \\
\%\end{array}$ & $\begin{array}{l}\text { GDP per } \\
\text { capita, } \\
\text { USD }\end{array}$ \\
\hline 1995 & $5,696,160,733$ & 1.47 & 30,828 & 5,372 & 7,495 & 2.86 & 1,316 \\
\hline 1996 & $5,777,240,510$ & 1.42 & 31,472 & 5,410 & 7,740 & 3.27 & 1,340 \\
\hline 1997 & $5,856,792,928$ & 1.38 & 31,358 & 5,319 & 8,027 & 3.7 & 1,371 \\
\hline 1998 & $5,934,983,696$ & 1.34 & 31,123 & 5,212 & 8,234 & 2.58 & 1,387 \\
\hline 1999 & $6,012,341,125$ & 1.3 & 32,305 & 5,339 & 8,511 & 3.37 & 1,416 \\
\hline 2000 & $6,089,661,543$ & 1.29 & 33,225 & 5,423 & 8,874 & 4.27 & 1,457 \\
\hline 2001 & $6,167,244,942$ & 1.27 & 33,049 & 5,328 & 9,038 & 1.85 & 1,466 \\
\hline 2002 & $6,245,056,652$ & 1.26 & 34,387 & 5,476 & 9,231 & 2.13 & 1,478 \\
\hline 2003 & $6,323,241,327$ & 1.25 & 38,656 & 6,081 & 9,495 & 2.86 & 1,502 \\
\hline 2004 & $6,401,941,947$ & 1.24 & 43,535 & 6,766 & 9,883 & 4.09 & 1,544 \\
\hline 2005 & $6,481,317,336$ & 1.24 & 47,153 & 7,240 & 10,240 & 3.61 & 1,580 \\
\hline 2006 & $6,561,466,555$ & 1.24 & 51,108 & 7,753 & 10,662 & 4.13 & 1,625 \\
\hline 2007 & $6,642,449,039$ & 1.23 & 57,520 & 8,622 & 11,084 & 3.96 & 1,669 \\
\hline 2008 & $6,724,189,737$ & 1.23 & 63,026 & 9,334 & 11,248 & 1.48 & 1,673 \\
\hline 2009 & $8,809,620,067$ & 1.23 & 59,803 & 8,752 & 11,022 & -2.01 & 1,619 \\
\hline 2010 & $6,889,628,242$ & 1.22 & 65,349 & 9,451 & 11,474 & 4.1 & 1,665 \\
\hline 2011 & $6,973,291,960$ & 1.21 & 72,374 & 10,345 & 11,798 & 2.83 & 1,691 \\
\hline 2012 & $7,057,203,973$ & 1.2 & 73,631 & 10,402 & 12,057 & 2.19 & 1,708 \\
\hline 2013 & $7,141,558,332$ & 1.2 & 75,566 & 10,553 & 12,331 & 2.27 & 1,727 \\
\hline 2014 & $7,226,178,654$ & 1.18 & 78,037 & 10,799 & 12,726 & 3.2 & 1,761 \\
\hline 2015 & $7,310,729,390$ & 1.17 & 73,502 & 10,054 & 13,107 & 3 & 1,793 \\
\hline 2016 & $7,397,835,935$ & 1.19 & 75,213 & 10,167 & 13,487 & 2.9 & 1,823 \\
\hline
\end{tabular}

It should be noted that we have a reduction in population growth almost in 2 times. If we compare GDP per capita to the population in 1970, then in 2016 GDP per capita should be USD 20,369.17. Another disadvantage of the calculation methodology is the lack of representation in GDP per capita of inequality in the incomes of economic agents and indicators of household indebtedness. This means that we can not account for the mismatch between the average income 
and the overall average income. For example, if the income of a certain segment of population will increase, then the average income in the country will increase, while the income of other segments of population may even decrease. We use market prices to evaluate goods and services. However, one can not rely on market prices because they are not converted in the current. Standard methods of measuring GDP are used in reports on the growth of a particular economy, but they do not show the indicators of household debt. Considering the latter could significantly correct the growth of economy, which is reflected in the reports of countries and international organizations.

\section{A new paradigm for assessing the public welfare of the national economic system}

Recent advances in the methodology have made it possible to assess the factors affecting the assessment of the well-being of citizens better, as well as to collect the data we need for analysis on a regular basis, in particular:

- loss of work characterizes not only the reduction of income, but also reflects a change in social status;

- quality of our lives should include sustainable development. Thus, firms estimate the impairment of their capital, but national economic complexes do not take into account the reduction of natural resources and degradation of the environment;

- the desire to increase GDP leads to neglect of the issue of security, the environment conditions, standards of life, etc.

We became convinced again that a modern alternative to GDP is needed. Today, there are two such indicators: The Social Progress Index and Inclusive Development Index. Let us consider them more comprehensively.

The Social Progress Imperative has developed the Social Progress Index (2014) in collaboration with Harvard Business School, Massachusetts Institute of Technology, as well as with leading international organizations in the field of social entrepreneurship, business and philanthropy. There are Cisco, Compartamos Bank, Deloitte Touche Tohmatsu Limited and its member companies, AVINA Foundation, Jeff Scoll's Fund, Sally Osberg, Steve Almond and Michael Porter among these.

The task of the Social Progress Imperative is to improve the welfare of people around the world, especially the poorest ones, by accelerating social development. The organization creates a reliable, single and innovative rating of social development; develops research and exchange 
of knowledge in the field of social development; provides managers and business professionals, authorities and citizens with new tools for policy and program implementation to achieve this goal. Social development is defined as the ability of society to meet the basic needs of its citizens and to create opportunities for improving the standard of living, as well as conditions for the full realization of potential of both individuals and groups of citizens.

The social development ranking uses the GDP per capita, which is calculated on the basis of the purchasing power parity (PPP) (the World Bank's statistics are collected). The international dollar has the same purchasing power to GDP, as the US dollar in the United States of America. GDP in accordance with PPP represents the sum of the gross product created by all residents in the economy, to which all taxes on goods are added and from which any subsidies that are not included in the cost of goods are deducted. It is calculated without taking into account deductions for depreciation or depletion of natural resources.

The social development rate focuses on indicators that take into account basic needs such as health care, education and human rights, social and environmental performance. Rating is a new tool that changes rules of the game; it is designed to provide great opportunities for governments, companies, social entrepreneurs and other community representatives, as well as to increase collective responsibility and identify opportunities for investment and economies of scale effect. This is the first rating in the world, which involves eliminating the dependence of the level of world social development of the country. The social development rating is designed to put together all the factors that determine social progress, evaluate the relevant indicators and determine the priority of the industry for the development of countries.

In the process of ranking compilation, the assessment of consequences was provided with benefits - expected life expectancy, literacy, freedom of choice, rather than the amount of public expenditures or adopted laws. Since social development rates are assessed separately from economic indicators in social development, this is the first time we are able to study the interdependence between economic and social development.

The level of social development is not a direct consequence of economic development. According to a new world ranking published by the American non-profit organization The Social Progress Imperative and presented at the Scoll World Forum on Social Entrepreneurship, economic growth does not always lead to positive changes in the social sphere.

Thus, the rating of social development shows that there is a direct relationship between economic indicators (calculated in GDP per capita) 
and the level of social development; the relationship between economic and social development varies with income growth; the economic development of low-income countries does not necessarily entail a significant increase in social development; a high level of GDP per capita contributes to the satisfaction of basic human needs, but does not guarantee the expansion of social opportunities. However, while incomes increase,»easy» achievements in the sphere of social development promoted by economic growth are almost exhausted, and further economic development leads to new social and environmental problems. For example, the indicator of environmental sustainability, which considers parameters such as greenhouse gas emissions, high-income countries are far ahead of low-income countries. Consequently, with the gradual development of economies oof low-income countries, the rate of environmental sustainability may worsen before it starts to show positive dynamics again. Most countries provide sufficient basic health care, access to education and knowledge level of adult population for their citizens. This may indicate that the Millennium Development Goals have positive impact on social progress in these spheres. In order to improve indicators such as personal security, higher education accessibility and environmental sustainability (with low results in many countries), countries may require the implementation of similar coordinated joint actions.

Inclusive Development Index. An alternative system for assessing the economic development of countries was discussed at the World Economic Forum (WEF) in Davos. It was called a more modern analogue of GDP. According to the WEF plan, the Inclusive Development Index should inform society and help sustained and inclusive economic development. This indicator takes into account not only GDP but also 11 other parameters, which means that it reflects the real state of affairs in the country more complexly, and not only its production capacity. The index is formed for the second year in a row.

The index includes 12 indicators, which are divided into 3 groups $^{7}$ :

1. Growth and development:

- GDP per capita (USD in value terms in 2010);

- labour productivity - GDP per one worker (USD);

- expected life expectancy (years);

- employment of the population (percentage);

2. Inclusiveness:

- the coefficient of stratification of society (from 0, without stratification, to 100);

\footnotetext{
${ }^{7}$ Zelenskiv M. V. Davose pridumali alternativu pokazatelyu VVP. I kto teper mirovoy lider? [Electronic resourcel / M. V. Zelenskiy // Meduza. — 2018. — Access mode: https://meduza.io/cards/v-davose-pridumalialternativu-pokazatelyu-vvp-i-kto-teper-mirovoy-

lider?utm_source=facebook.com\&utm_medium $=$ share_fb\&utm_campaign=share.
} 
- poverty rate (percentage);

- coefficient of stratification of society by distribution of wealth (from 0, without stratification, to 100);

- median income (USD);

3. Continuity of generations and sustainability of development:

- adjusted net savings (percent of gross national income);

- the parity intensity of GDP (kilograms of CO2 emissions per USD);

- government debt (percent of GDP);

- dependency ratio (percent).

Thus, we can confidently confirm that alternative indicators should be calculated on the basis of those, which will reflect the effectiveness of the functioning of the country's economic system in terms of improving the well-being of every person and national prosperity in general more effectively.

So, in 2008 French President N. Sarkozy has created an international commission on changes in key indicators of economic activity and social progress due to the unsatisfactory state of statistical information in the economy and society. Steve Almond, chairman of the international board of directors of Deloitte Touche Tohmatsu Limited, also noted that a new indicator of social development should be considered in order to achieve progress. Sally Osberg, president and executive director of the Jeff Scoll Foundation, said that in order to receive the top priority of social development, knowledge of the development of society and its well-being to the value of GDP should be equated. Stuart Wells suggested using five indicators of economic growth (well-being, good jobs, environment, justice and health) instead of $\mathrm{GDP}^{8}$. Akshay Mole proposed to consider the Human Development Index, which takes into account the Life Expectancy Index, Education Index and Income Index instead of GDP ${ }^{9}$. Duncan Geere suggested to consider GNI per capita, the Human Development Index, the Happiness Index, the Gini coefficient, the Social Progress Index instead of GDP ${ }^{10}$. David Owen, Managing Partner of Deloitte in Russia, said that there is an opportunity to improve the well-being of the population in countries with developing economies: health and safety should be the priority sectors.

\footnotetext{
${ }^{8}$ Raynert E. S. Kak bogatyie stranyi stali bogatyimi, i pochemu bednyie stranyi ostayutsya bednyimi [Electronic resource] / per. s angl. N. Avtonomovoy; pod red. V.Avtonomova; Gos. un-t - Vyisshaya shkola ekonomiki. - M.: Izd. dom Gos. un-ta — Vyisshey shkolyi ekonomiki, 2011. — 384 p. — Access mode: http://www.uhimik.ru/how-richcountriesgot-rich-and-why-poor-countries/index5.pdf [In Russian]

Bondarchuk $V$. Chomu pokaznyk VVP ne vidpovidaie suchasnym potrebam vymiriuvannia ekonomichnoho zrostannia ta shcho maie pryity yomu na zminu [Electronic resource] / V.Bondarchuk // Analitychna platforma VoxUkraine - Access mode: https://archive.voxukraine.org/2017/01/04/economic-growth-ua/. [In Ukrainian]

${ }^{10}$ Duncan G. GDP Sucks. Here Are Some Better Alternatives [Electronic resource]/ Geere Duncan // Next. 2016. - Accessed: https://howwegettonext.com/gdp-sucks-here-are-some-better-alternatives- $88 \mathrm{cf} 2 \mathrm{bfec} 017$.
} 
However, most of the proposed indicators can not be an alternative to GDP. For example, the Education Index takes into account the number of people with a degree in education, but does not take into account the quality of education in the country. Indices of social progress and happiness are based only on the questionnaires. The national income per capita and Gini coefficient are based on statistical information that does not take into account the shadow economy and shadow income of the population. In case of choosing a false analysis instrument, one should reflect on the result of the analysis. For example, in the process of production of machinery, there was no increase in the number that affects GDP, but quality implementation was introduced. The society is provided with a large number of medical services, but most of their achievements relate to quality improving. Thus, the United States is spending more on health care than any other country, but it gets less effectiveness (relation to per capita, percentage expression to income).

\section{Conclusions}

Welfare of population is the ultimate goal of any economic system, including the market. The level of social welfare is evaluated on the basis of many criteria, both quantitative and qualitative. However, since the Second World War, GDP was the leading indicator, which was conditioned by the need to develop an indicator of aggregate activity. It should be noted that the founder of this methodology, the Nobel laureate in economics Simon Kuznets considered this approach unlawful. In his view, the purpose of changing the national product was the need to assess individual parts of this indicator (production system) and their response to various types of stimulation ${ }^{11}$.

Consequently, given the initial existence of the defects of this macroeconomic indicator, it continues to be used today as the main measure of economic prosperity, which requires a further critical rethinking from several points of view. Firstly, it is advisable to take into account indicators outside the sphere of money circulation, which characterize the state of the environment and social maturity of society. So, even in the second half of the last century, the report of the Club of Rome made another attempt to draw attention to the deterioration of the ecological state (for example, maintaining the viability of the oceans and the atmosphere, the ability of trees to clean the air, etc.), which will require further increase in costs due to the profits, causing

\footnotetext{
${ }^{11}$ Kuznets S. National Income, 1929-1932 / Simon Kuznets. // Senate document, 73rd US Congress, 2d session. - 1934. - No.124. - pp. 7-9.
} 
reduction of public welfare. Another component that is not included in the social welfare is social well-being. In particular, the system of national accounts have no record of distributed income (social inequality), since the wealth of one part of the population conceals the poverty of another; manufactured goods and / or services provided by households are not reflected in GDP in all national economies, and so on. Secondly, the lack of accounting in the national income of the shadow economy, which plays a more important role in underdeveloped economic systems. Thirdly, digitalization of the global economy is affecting the change in the working methods of a large number of people, which erases the boundaries between home and work. People are increasingly self-employed or working on a freelance basis, using digital platforms. Their working hours can be flexible, and their primary work may be partly combined with other activities. In many cases, they use the assets of their households for paid work - from computers and smartphones to their own homes and cars. Many people work free of charge digitally, developing open source software that can replace its pay-equivalents and, despite zero price, clearly has a significant economic value.

Evolutionary changes in the development of the world economic system sharpen the need to develop an indicator that will become the successor of GDP in determining social welfare. Currently, such an alternative is social and inclusive development indicators. Alternative indicators include all components of social welfare, which allows us to trace the correlation between its components (economic and social wellbeing). However, despite the relative progressiveness of the new indicators, they are not free from certain disadvantages. In particular, the exhaustion of the resource potential, which contributes to the development of the social sphere of the country, further development of the economy leads to the emergence of new environmental and social problems. Thus, the imperfection of old and new indicators of public welfare assessment has somehow similar problems that make expediency to continue research. Thus, change in the paradigm of socio-economic system and approaches to its evaluation from purely economic to socioeconomic (public) welfare.

\section{References}

1. Andreeva E. H. Ekonomycheskyi rost. Modely Ekonomycheskoho rosta / E. H. Andreeva, A. N. Sukhova // Omskyi nauchnyi sotsyolohychesky y ekonomycheskye nauky vestnyk. - 2011. - No. 6 (102). - pp. 46-50. [In Russian]

2. Bondarchuk V. Chomu pokaznyk VVP ne vidpovidaie suchasnym potrebam vymiriuvannia ekonomichnoho zrostannia ta shcho maie pryity yomu na zminu 
[Electronic resource] / V.Bondarchuk / / Analitychna platforma VoxUkraine Access mode: https://archive.voxukraine.org/2017/01/04/economic-growth-ua/. [In Ukrainian]

3. Buchanan M. How Technology Might Get Out of Control [Electronic resource] M. Buchanan // Bloomberg. - 2017. - Access mode: https://www.bloomberg.com/ opinion / articles / 2017-08-15/how-technology-might-get-out-of-control

4. Comparison of Nominal and Real GDP and GDP per capita / Statistics Times [Electronic resource]. - Access mode: http://statisticstimes.com/economy/gdpnominal-vs-gdp-ppp.php

5. Countries by Projected GDP / Statistics Times [Electronic resource]. - Access mode: http: / / statisticstimes.com/economy / countries-by-projected-gdp.php

6. Duncan G. GDP Sucks. Here Are Some Better Alternatives [Electronic resource] / Geere Duncan // Next. - 2016. - Accessed: https://howwegettonext.com/gdpsucks-here-are-some-better-alternatives-88cf2bfec017.

7. Economic forecast «The World in 2050»/PwC [Electronic resource]. - Access mode: https: / / www.pwc.com/ua/uk/press-room/2015/the-world-in-2050.html

8. GDP / World Bank [Electronic Source]. - Access mode: https: / / data.worldbank.org/indicator/NY.GDP.MKTP.CD

9. Ivaskiv I. Staraya novaya piramida Maslou ili V chyom izmeryaetsya schaste? [Electronic resourse] // Life Academy. - 2018. - Access mode: http: / / lifeacademy.pearl.pp.ua/staraja-novaja-piramida-maslou-ili-v-chjomizmerjaetsja-schaste.html. [In Russian].

10. Kuznets S. National Income, 1929-1932 / Simon Kuznets. / / Senate document, 73rd US Congress, $2 d$ session. - 1934. - No.124. - pp. 7-9.

11. Litvinyuk A.A. Motivatsiya i stimulirovanie trudovoy deyatelnosti. Teoriya i praktika: uchebnik dlya bakalavrov [Electronic resource] / A. A. Litvinyuk. Moskva: Izdatelstvo Yurayt, 2015. - 398 p. - (Seriya: Bakalavr. Akademicheskiy kurs). - Access mode: https://stud.com.ua/34677/menedzhment/motivatsiya_i_ stimuly [In Russian].

12. Lytovchenko T. Epokha rivnovahy Nesha zakinchuietsia? [Electronic resource] / T. Lytovchenko // Chas $i$ podii. - 2017. - No.35. - Access Mode: https://www.chasipodii.net/article/19403/. - Режим доступу до ресурсу: https: / / www.chasipodii.net/article/19403/ [In Ukrainian].

13. McConnell K. Economics / K. McConnell., 2017. - 984 c. - (21). (Series in Economics).

14. Mizhnarodna ekonomika: pidruchnyk / [A. P. Rumiantsev, H. N. Klymko, V. V. Rokocha ta in.]. - [Vyd. pererobl. i dopov.]. - Kyiv: Znannia, 2006. - 480 p.

15. Mochernyi S.V. Ekonomichna teoriia: pidruchnyk [Elektronnyi resurs] S. V. Mochernyi, M. V. Dovbenko. - K.: Akademiia, 2005. - 856 p. - (Alma mater). - Access mode: //http://pidruchniki.com/ekonomika/rivnovaga_nesha [In Ukrainian].

16. Natsionalna ekonomika: navch. posib. [Electronic resourse] / A. F. Melnyk, A. Iu. Vasina, T. L.Zheliuk, T. M.Popovych; za red. A. F.Melnyk. - K.: Znannia, 2011. - 464 p. - Access mode: / / http://pidruchniki.com/1298010841794/ekonomika/ kontseptsiyi_efektivnosti_konkurentnoyi_rinkovoyi_ekonomiki [In Ukrainian].

17. Raynert E. S. Kakk bogatyie strañyi stali bogatyimi, $i$ pochemu bednyie stranyi ostayutsya bednyimi [Electronic resource] / transl. from Eng. N. Avtonomovoy; pod red. V.Avtonomova; Gos. un-t - Vyisshaya shkola ekonomiki. - M.: Izd. dom Gos. un-ta - Vyisshey shkolyi ekonomiki, 2011. - 384 p. - Access mode: 
http: / / www.uhimik.ru / how-richcountriesgot-rich-and-why-poor-countries /index5.pdf [In Russian]

18. Real GDP Growth / International Monetary Fund [Electronic resource]. Access mode: http://www.imf.org/external/datamapper/NGDP_RPCH@WEO/ OEMDC / ADVEC /WEOWORLD? year=2018

19. Robert F. Kennedy speeches at the University of Kansas [Electronic resource]. - 18 march 1968. - Accessed: https://www.jfklibrary.org/learn/about-jfk/thekennedy-family / robert-f-kennedy / robert-f-kennedy-speeches / remarks-at-theuniversity-of-kansas-march-18-1968

20. Rodionov-Zrazhevskiy A. G. Ot epohi valovogo vnutrennego produkta k «ekonomike schastya» / A. G. Rodionov-Zrazhevskiy / / Problemyi sovremennoy ekonomiki. - 2013. - \# 3 (47). - pp. 129-131. [In Russian]

21. Shyian A.A. Teoriia ihor: osnovy ta zastosuvannia $v$ ekonomitsi ta menedzhmenti. Navchalnyi posibnyk [Elektronnyi resurs]. - Vinnytsia: VNTU, 2009. - 164 p. - Access mode:: http://inrtzp.vntu.edu.ua/pmba/stf/teach/books / Theory.pdf [In Ukrainian].

22. World Economic Outlook. Cyclical Upswing, Structural Change [Electronic resource] / / International Monetary Fund. - 2018. - Accessed: https: / / www.imf.org/en / Publications /WEO/Issues / 2018/03/20/worldeconomic-outlook-april-2018

23. Zelenskiy M. V Davose pridumali alternativu pokazatelyu VVP. I kto teper mirovoy lider? [Electronic resource] / M. V. Zelenskiy //Meduza. - 2018. Access mode: https://meduza.io/cards/v-davose-pridumali-alternativu-pokazatelyuvvp-i-kto-teper-mirovoy-lider?utm_source=facebook.com\&utm_medium=share_fb\&utm_ campaign=share. [In Russian]

The article was received by the editorial board on 13.03.2019. 